\title{
Editorial
}

\section{Acknowledgement to Reviewers of the International Journal of Environmental Research and Public Health in 2013}

IJERPH Editorial Office, MDPI AG, Klybeckstrasse 64, CH-4057 Basel, Switzerland

Published: 28 February 2014

The editors of the International Journal of Environmental Research and Public Health would like to express their sincere gratitude to the following reviewers for assessing manuscripts in 2013:

\begin{tabular}{lll} 
Aasvang, Gunn & Amaro, Ines & Aslan, Asli \\
Abballe, Annalisa & Amin, Raid & Atsuo, Kishimoto \\
Abreu, Mauro & Anafi, Patricia & Attanasio, Laura \\
Abughosh, Susan M. & Ancona, Nicola & Attfield, Michael \\
Abuknesha, Ramadan A. & Andersen, Helle R. & Auchincloss, Amy H. \\
Acharya, Lalatendu & Andersen, Lars L. & Aumann, Craig \\
Adams, James B. & Anderson, Peter & Aung, May \\
Adams, Monica L. & Anderson, Peter D. & Aunger, Robert \\
Adeseun, Gbemisola & Anenberg, Susan C. & Aurrekoetxea, Juan J. \\
Adlam, Timothy D. & Añez, Germán & Austin, Laurel \\
Adler, Meghan E. & Angelopouloua, Elli & Autenrieth, Christine \\
Afonso, José A. & Anopa, Yulia & Avogo, Winfred Aweyire \\
Agalakova, Natalia & Antunes, Patrícia & Ayo-Yusuf, Olalekan A. \\
Aittasalo, Minna & Appel, Wyat & Azmitia, Efrain C. \\
Akhalkatsi, Maia & Ara, Ignacio & Babalola, Stella O. \\
Albert, Steven M. & Arboleda, Sair & Backhans, Annette \\
Alencar, Carlos Henrique & Arcury, Thomas A. & Bagella, Simonetta \\
Allanson, Paul & Argos, Maria & Bagramian, Robert \\
Allen, Harris & Arimilli, Subhashini & Baker, Mary Kate \\
Allerberger, F. & Ariño, Cristina & Bakonyi, Tamas \\
Altaweel, Mark & Armstrong, Philip M. & Bal, Wojciech \\
Alverson, Dale C. & Arroyo-Cobo, Jose Manuel & Balcazar, Hector \\
Alves, Elisabete & Asakawa, Akihiro & Balk, Sophie J. \\
Amarasena, Najith & Aslam, Mueen & Ballbè, Montse \\
\hline
\end{tabular}


Bammann, Karin

Banach, Maciej

Bannon, Desmond

Bao, Yongping

Barber, Sarah L.

Barbieri, Flavia

Barclay, Kieron

Barlow, Karen Maria

Barnes, Philip L.

Barnett, Tracey E.

Barreca, Alan

Barrera, Roberto

Bartold, Mark

Barzon, Luisa

Bashshur, Rashid L.

Basu, Rupa

Batchelor, Frances

Baumgarth, Nicole

Baverstock, Keith

Bayat, Fariborz

Bayer, Ronald

Beaver, Kevin M.

Becoña, Elisardo

Bedore, Melanie

Beebe-Dimmer, Jennifer

Beenackers, Marielle A.

Befort, Christie

Behson, Scott J.

Beilhe, Leila Bagny

Belasco, Eric

Beldowski, Jacek

Bellatreche, Ladjel

Bellomini, Raffaella

Bellou, Andriana

Benbenishty, Rami

Benfield, Jacob

Benjamin-Garner, Ruby

Bennett, Jamie

Bere, Elling

Berg, Christine D.

Berntsen, Sveinung

Berry, E. Helen
Berry, Peter

Bertherat, Eric

Berwick, Marianne

Bett, Bernard

Bhandari, Medini

Bhunia, Arun

Bianchi, Fabrizio

Bierman, Karen

Bingham, John

Bisson, Mary A.

Biziuk, Marek

Björkenstam, Emma

Blanco, Cristina

Blanford, Justine

Blank, Michael

Blitvich, Brad

Bloch, Michele

Blunt, Ruth

Bobylev, Nikolai

Bocchiccio, Francesco

Bockerman, Petri Rikhar

Bonevski, Billie

Boniface, David

Boot, Cécile

Bopp, Melissa

Borchard, Mark A.

Borja-Aburto, Víctor Hugo

Boroushaki, Mohammad Taher

Borse, Nagesh N.

Bostelman, Roger

Boudrez, Hedwig

Boyce, Walter

Boyd, Robert S.

Bradstreet, James Jeffrey

Brand, Serge

Brandsema, Petra

Braubach, Matthias

Brault, Aaron C.

Breitner, Susanne

Brenner, Ingrid

Briggs, David

Brink, Mark
Broadbent, Jonathan M.

Brodie, Ian M.

Bronsvoort, Mark

Brooks, Antone

Brough, Helen

Brown, Helen

Browne, Marilyn

Brunette, Mary

Brunetti, Virgilio

Brussoni, Mariana

Buckwalter, J. Galen

Budke, Christine M.

Bugiardini, Raffaele

Buja, Alessandra

Bujnowska-Fedak, Maria

Magdalena

Burd, Larry

Burd, Martin

Burdorf, Alex

Burk, Tonia

Burrough, Eric

Burrows, Stephanie

Bus, Boudewijn A. A.

Bush, Terry

Busova, Milena

Butler, Danielle C.

Byass, Peter

Byrd-Bredbenner, Carol

Byrne, Dominic

Cabral, João P. S.

Cai, Bo

Calderaro, Adriana

Calderón-Garcidueñas, Lilian

Calistri, Paolo

Calvert, Amanda E.

Cama, Jordi

Campbel, Laura

Campbell, Daniel

Campbell, Kathleen C. M.

Campos, Guillermo Lopez

Can, Arnaud

Canfield, Mark A. Canfield 
Cantón-Cortés, David

Cao, Kai

Capra, Valeria

Caputo, Tullio

Capuzzo, Maurizia

Cardel, Michelle

Caroli, Sergio

Carpenter, David

Carpentier, Brigitte

Carrera, María Victoria

Carrero, Jose Antonio

Carrieri, Marco

Carrieri, Vincenzo

Carson, Kristin

Carter, Megan A.

Carvajal, Scott

Cassidy-Bushrow, Andrea E.

Casson, Karen

Castellano, Immacolata

Chagnes, Alexandre

Chaiton, Michael

Chan, Ding-Cheng

Chandwani, Sulachni

Chang, Ching-Cheng

Chang, Hung-Hao

Chari, Ramya

Chariot, Patrick

Charles, Katrina

Charlton, Brittany M.

Chatignoux, Édouard

Chatzipanagiotou, Stylianos

Chau, Chi-Kwan

Chaves, Luis Fernando

Chen, Chi-Feng

Chen, Keping

Chen, Kow-Tong

Chen, Lei

Chen, Pei-Shih

Chen, Szu-Chieh

Chen, Wan-Qing

Chen, WeiHong

Chen, XinGuang
Chen, Zhuo

Cheong, Hae-Kwan

Cherrington, Nathan J.

Cheung, Chau-Kiu

Chiabai, Aline

Chiara, Panari

Chilenski, Sarah M.

Chin, Dal Lae

Chiou, Hung-Yi

Chipps, Jennifer

Chirizzi, Daniela

Cho, Kyoung-Im

Chobert, Jean-Marc

Choi, Kelvin

Chomistek, Andrea K.

Chomutare, Taridzo

Chou, Yiing-Jeng

Chow, Maria Yui Kwan

Chow, Winston T. L.

Christensen, Henrik

Christophe, Daniel

Christophe, Waterlot

Chuang, Chun-Yu

Chuang, Kai-Jen

Chuang, Ting-Wu

Chung, Chung-Yi

Chung, Stephan

Ciccozzi, Massimo

Cikajlo, Imre

Claes, Neree

Claeson, Anna-Sara

Clarke, Malcolm

Clarkson, Peter M.

Clayton, Susan

Cliff, David

Clougherty, Jane E.

Cocco, Pierluigi

Cochran, Joshua C.

Coelho, Ana Cláudia

Cohen, Art

Cohen, Mitchell D.

Cohen, Samuel M.
Coldea, Gheorghe

Coleman, Brenda

Collings, Paul

Collins, Marietta H.

Collins, Timothy W.

Colwell, Doug

Congdon, Peter

Conger, Scott

Cook, Joseph H.

Cooper, David

Cooper, Ralph

Cordioli, Paolo

Cormac, Irene

Cormier, Yvon

Cornelsen, Laura

Coronato, Antonio

Coss, Richard

Costa, Max

Costea, Daniela Elena

Coulton, Claudia J.

Cox, Chad

Cox, Georgina

Coyne, Mark

Crane, Patricia

Croezen, Simone

Cronkite, Ruth C.

Cruz-Mendoza, Irene

Cruz, Adriano G.

Culberson, Kurt

Cullinan, Paul

Cummings, David E.

Currid, Thomas

D'anna-Hernandez, Kimberly

D'Arcy, Shona

Da Cunha, Moore K. Dias

Dai, Heping

Dakulincová, Zuzana

Dale, Ann

Dalton, Alice M.

Dalton, Harry R.

Damashek, Amy

Damiani, Gianfranco 
Danielsen, Tor Erik

Darbra, Rosa-Mari

Davies, Robert

Davis, Mark

Davis, Meghan F.

De Carvalho Ponce, Julio

De Coensel, Bert

De França, Elvis Joacir

De Grey, Aubrey

De Haan, Caroline

De Hert, Marc

De Hert, Paul

De La Torre Reoyo, Ana

De Leo, Gianluca

De León, Byron Calgua

De Luca, Patrick

De Munter, Jeroen

De Oliveira Fernandes,

Eduardo

De Sario, Manuela

De Souza Vieira, Luiza Jane

Eyre

Debess, Emilio E.

Decker, Sandra L.

Del Razo, Luz

Demeke, Tigst

Demiroglu, Cenk

Desenclos, Jean-Claude

Deserno, Thomas M.

Devís-Devís, José

Devleesschauwer, Brecht

Devlieger, Roland

Dewan, Ashraf

Dewe, Philip

Dickerson, Faith

Difranza, Joseph

DiGiacomo, Michelle

Diraco, Giovanni

Dirks, Kim N.

Dismuke, Libby

Dittmer, Dirk

Dittrich, Ralf
Diuk-Wasser, Maria A.

Divaris, Kimon

Djenane, Djamel

Djietror, Godwin

Djomo, Sylvestre Njakou

Do, D. Phuong

Dobke, Marek K.

Dockery, Michael

Doctor, Henry

Dodson, Robin

Domínguez, Angela

Dong, Cheng-Di

Dong, Xin Qi

Dons, Evi

Dorevtich, Sam

Douglas, Ian

Douterelo, Isabel

Du, Hongfei

Du, Libo

Dubrova, Yuri E.

Ducret-Stich, Regina E.

Duffy, Sonia A.

Dumètre, Aurélien

Dunbar, James D.

Dunbar, Michael Stephen

Dunière, Lysiane

Dunn, Gemma

Dunst, Carl J.

Dupuis, Alan

Durey, Angela

Durrani, Hammad

Dykman, Lev A.

Easterling, Doug

Ebi, Kristie

Eckhart, Vincent M.

Edwards, Ben

Egli, Thomas

Eichner, Martin

Eisenberg, Daniel

Eisenberg, Joseph

Ekman, Björn

Elenge, Myriam
Elizur, Arnon

Elliott, Michael B.

Elstad, Jon Ivar

English, Paul

Erazo, Marcia

Erhart, Laura

Erreygers, Guido

Escobedo, Francisco J.

Escoffery, Cam

Evans, Ken

Eves, Frank

Eysel-Gosepath, Katrin

Fabian, Patricia

Fagan, Abby

Fallin, Amanda

Fang, Chen-Ling

Fang, Hui

Fann, Neal

Farr-Wharton, Rod

Faruque, Fazlay

Federico, Bruno

Felföldi, Tamas

Feng, Peter

Fensli, Rune

Fergusson, David M.

Fernandez-Ballesteros, Rocío

Ferrario, Marco M.

Ferro, Roberto

Fesus, Gabriella

Fickenscher, Helmut

Figueira, Etelvina

Finch, Meghan

Finkelstein, Fedric

Finlayson, Janet

Finley, Richard

Fischer, Arnout

Fischer, Dominik

Fischer, Gloria

Fischer, Joachim E.

Fisher, Gwenith G.

Fisk, Malcom J.

Flemming, Hans-Curt 
Fleury, Anthony

Florence, Curtis S.

Fonnum, Frode

Fonseca, Dina M.

Font, Laia

Ford, Timothy

Fornalski, Krzysztof Wojciech

Fornaro, Adalgiza

Forsberg, Bertil

Forsythe, Seteve

Foster, John

Fostervold, Knut-Inge

Foubert, Imogen

Fozard, James L.

Frank, Andrew

Franklin, Richard

Fransson, Eleonor

Franzblau, Alfred

Franzetti, Laura

Frederick, Tyler

Freeman, Matt

Freeman, Toby

Freestone, Mark

Freiberger, Ellen

Frenken, Melina

Friesen, Kristina M.

Froehlich-Grobe, Katherine

Frohlich, Katherine

Frye, Richard E.

Fuchs, Regina

Fujii, Yasuhito

Fuller, Richard

Furgal, Christopher

Fustinoni, Silvia

Gagnon, Marie P.

Gaines, David

Galani, Irene

Gall, Seana

Gallagher, Carolyn

Gallagher, Carolyn M.

Galve, Roger

Gamble, John F.
Gao, Chuansi

Gao, Xubo

Garin, Benoit

Garrett, Sue

Gary, Faye

Gašević, Danijela

Gates, Michael G.

Gaudart, Jean

Gautier, Axel

Gbaguidi-Haore, Houssein

Gebreab, Samson

Geller, E. Scott

Genchi, Claudio

Généreux, Mélissa

George, David T.

Giatropoulos, Athanassios

Gibson, Chris L.

Gibson, Maggie

Giddon, Donald B.

Gidlöf-Gunnarsson, Anita

Giesbrecht, Norman

Gikas, Petros

Gilbert, Paul

Giles, Audrey

Gili, Margarida

Gilkey, David P.

Gill, Jan S.

Gillings, Michael

Gilroy, Rose

Giné Garriga, Ricard

Giraud, Etienne

Gislason, Maya

Gissler, Mika

Glaesmer, Heide

Glockner, Karl

Glynn, Peter J.

Goarant, Cyrille

Goldstein, Adam

Gomez, Pilar Garcia

Gonçalves, Guilherme

Goonetilleke, Ashantha

Goovaerts, Pierre
Gordon, Kathryn

Gore, Andrea

Gore, Dana

Gorini, Giuseppe

Gosselin, Perre

Govaris, Alexander

Grabrucker, Andreas

Gradoni, Luigi

Grandadam, Marc

Grant, Marcus

Grant, Robert

Grant, William B.

Granum, Per Einar

Graudal, Niels Albert

Gray, Selena

Greacen, Tim

Greene, Nicholas

Greene, Scott

Grigoriev, Pavel

Grineski, Sara

Grittner, Ulrike

Grooten, Wim

Groschup, Martin

Grossi, Enzo

Grundstein, Andrew J.

$\mathrm{Gu}$, Jianwei

Guernsey, Judith

Guirguis, Kristen

Gullo, Maria

Guo, Jong-Long

Gupta, Amar

Gupta, Jhumka

Gushulak, Brian D.

Gustafsson, Susanne

Guyer, Bernard

Ha, Sang-do

Haber, Steve

Hadjichristodoulou, Christos

Hagen-Zanker, Alex

Hager, Erin R.

Hahn, Joan

Hahn, Youjin 
Hailey, David

Hale, Leigh

Hall-Mendelin, Sonja

Hall, Pamela R.

Halonen, Jaana I.

Halperin, William E.

Halsall, Jamie

Hambach, Ramona

Hamer, Gabriel L.

Hamer, Mark

Hammitt, James

Hampson, David J.

Hancock, Trevor

Hanewinkel, Reiner

Hanke, Wojtek

Hanna, Liz

Hansen, Claus D.

Hanson, Timothy

Harada, Masafumi

Harding, John

Hariharan, Harry

Harjutsalo, Valma

Harlan, Sharon

Harley, David

Harony, Hala

Harper, Sherilee

Harrington, John

Harris, Anne M.

Harris, Matthew

Hartig, Terry

Harvey, Roger

Hashsham, Syed A.

Haslam, Divna M.

Hasselberg, Marie

Hauer, Klaus

Haukka, Kaisa

Hawley-Hague, Helen

Hayes Jr., Don

$\mathrm{He}$, Jie

He, Yonghua

He, Yongqing

Healy, Genevieve N.
Heaviside, Clare

Heilig, Charles M.

Hein, Andreas

Hendrickx, Guy

Hendryx, Michael

Henkin, Alan B.

Hennrikus, Deborah

Henriksen, Lisa

Hens, Niel

Heo, Jinseok

Heo, Jongho

Hernandez-Jover, Marta

Hernando Sebastián, Victoria

Heukelbach, Jorg

Hines, Cynthia J.

Hirsch, Alec J.

Hiscock, Rosemary

Hitzhusen, Frederick

Ho, Sai Yin

Hobst, Leonard

Hochrainer-Stigler, Stefan

Hoff, Dale J.

Hofmann, Bjørn

Hofmeister, Erik K.

Hogan, Anthony

Holbrook, Michael R.

Holick, Michael F.

Hollen, Vera

Holzel, Christina

Hondula, David

Hong, Jun Sung

Hooker, Brian

Hoppin, Jane

Hoppin, Polly

Hornikx, Maarten

Horton, Graeme

Hosgood III, H. Dean

Hoshiko, Sumi

Hoskins, John A.

Houghton, Adele

Howard, Matthew O.

Howe, Peter
Hoyert, Donna L.

Hrubá, Drahoslava

Hsiao, Hui-Wen

$\mathrm{Hu}$, Brian

$\mathrm{Hu}$, Xuefei

Huang, Gordon

Huang, Jin

Huang, Jiun-Hau

Huang, Lihua

Huang, Wei-Shin

Hudson-Edwards, Karen

Huen, Karen

Hughes, Karen

Huijts, Tim

Hunt, Alistair

Hunter, Bronwyn A.

Hunter, Ruth

Huntington, Henry

Hurme, Markku

Hutchinson, Amanda

Hwang, Cheng-An

Hyder, Adnan A.

Hystad, Perry

Iamarino, Mario

Ianieri, Adriana

Ichiba, Masayoshi

Ikem, Abua

Jacinto, Celeste

Jacobs, David

Jahnke, Sara A.

Jakovljevic, Mihajlo

Jakubovics, Nicholas S.

Jakus, Paul

Jalava, Katri

Jambrosic, Kristian

Janczukowicz, Wojciech

Jang, Hyung-Kwan

Janson, Staffan

Jardine, Andrew

Jawad, Mohammed

Jay, Ollie

Jennett, Penny 
Jeon, Byeonghwa

Jeon, Jin Yong

Jeong, Hyo-Joon

Jeong, Seok Hoon

Jepsen, Martin Rudbeck

Jesdale, Bill

Jetter, James J.

Jevšnik, Mojca

Jex, Aaron R.

Jiang, Hongchen

Jiang, Yu

Jimenez-Cisneros, Blanca

Jiménez, Víctor Segura

Jing, Chuanyong

Jo, Wan-Kuen

Johansen, Cheryl

Johansson, AnnaKarin

Johansson, Per

John, Dolly A.

Johnson, Aaron

Johnson, Brian J.

Johnson, Greg

Johnson, Linda S. B.

Johnston, Carl Gordon

Johnston, Douglas M.

Johnston, Jill E.

Jones, Anne

Jones, Ray

Jönsson, Kristina

Jordan-Marsh, Maryalice

Jorstad-Stein, Ellen C.

Joubert, Karin

$\mathrm{Ju}$, Hwang Won

Julian, Ernest

Jun, Young-Shin

Jung, Myung-Chae

Jurewicz, Joanna

Kaang, Bong-Kiun

Kahan, David

Kaiiry, Dahlia

Kalengayi, Faustine Nkulu

Kaltenbach, Martin
Kamp, David W.

Kampel, Martin

Kang, Choong-Min

Kang, Sanghoon

Kang, Yanming

Karakitsios, Spyros

Karanikolos, Marina

Karanis, Panagiotis

Karlsson, Martin

Käsbohrer, Annemarie

Kasper, Christine

Katanoda, Kota

Katayama, Hiroyuki

Katbamna, Bharti

Katner, Adrienne

Kaufman, James S.

Kawamura, Takashi

Kayhanian, Masoud

Keener, Tim

Keeshin, Brooks

Kefauvera, Shawn C.

Keizer, Ineke

Kelepertzis, Efstratios

Kemptner, Daniel

Kerminen, Veli-Matti

Kern, Janet K.

Kerret, Dorit

Kershaw, Kiarri N.

Kesavaraju, Banugopan

Kessler, Ronald C.

Key-Schwartz, Rosa

Khan, Arifulla

Kiem, Anthony

Kile, Molly

Kim, Heon

Kim, Ho

Kim, Hun Kyung

Kim, Hyun

Kim, Jeungim

Kim, Ki-Hyun

Kim, Sun S.

Kimura-Ono, Aya
King, Eoin

Kipen, Howard M.

Kira, Mari

Kirkpatrick, Sharon

Kishida, Naohiro

Kjellsson, Gustav

Klaschka, Ursula

Klein, Elizabeth

Kleinsteuber, Sabine

Klimas, Jan

Knobf, M. Tish

Knopper, Loren

Ko, Jiunn-Liang

Koehly, Laura M.

Koelemay, Mark J. W.

Kogut, Michael H.

Koivisto, Joonas

Kolehmainen, Mikko

Koletsi-Kounari, Haroula

Kolovos, Alexander

Komar, Nicholas

Kominkova, Dana

Kong, In Chul

Konishi, Eiji

Koo, Malcolm

Koohsari, Javad

Korfmacher, Katrina

Kormas, Konstantinos

Korsak, Dorota

Korzeniewska, Ewa

Kosterink, Stephanie

Kostkova, Patty

Kostova, Deliana

Kotzalidis, Giorgio D.

Koufi, Vassiliki

Kousoulas, Konstantin G.

Kovalenko, Kaspars

Kovalskyy, Valeriy

Kozyrskyj, Anita L.

Krakat, Niclas

Kramer, Laura D.

Kranz, Ashley M. 
Kreichauf, Susanne

Krienert, Jessie L.

Kristiansen, Jesper

Kristjuhan, Ülo

Kroesen, Maarten

Krogsbøll, Lasse T.

Krupinski, Elizabeth

Krysinska, Karolina

Kudirkienè, Eglè

Kühner, Dietrich

Kukizaki, Masato

Kulesza Jr., Randy J.

Kull, Inger

Kundi, Michael

Kungskulniti, Nipapun

Kuo, Shu-Chen

Kvaavik, Elisabeth

Kwapisz, Agnieszka

Lacerda, Silvia H.

Lachmann, Nico

Ladeau, Shannon L.

Lagrosen, Yvonne

Lai, Chih-Ho

Lai, Dejian

Lai, Ka-Man

Lamb, Joseph

Lamm, Steven H.

Lämmle, Lena

Lampman, Rich

Lando, Amy

Lando, Harry

Landschoot, Sofie

Lang, Katrin

Langley, Kate

Lapeyrouse, Lisa

Lassnig, Heimo

Lau, Michael

Laurent, Éloi

Laurier, Dominique

Lavelle, Bridget

Lavoué, Jérôme

Lawn, Sharon
Lawson, Andrew B.

Layte, Richard

Lazos, Evangelos S.

Lazuras, Lambros

Le Strat, Yann

Leal, Luz O.

Leão, Pedro N.

Lee, Duk-Hee

Lee, Hu Jang

Lee, Jeong-Chae

Lee, Joseph G. L.

Lee, Kylie

Lee, Lisa M.

Lee, Sue Ann S.

Lee, Won Jin

Lee, Won-Jeong

Lehucher-Michel, Marie-

Pascale

Lei, Yunping

Leicester, Andrew

Leisner, Jorgen J.

Lemke, Klaus

Lenz, Bernd

Leon, Natalie

Leong, David

Lepak, Jesse

Leslie, Timothy F.

Lessman, Charles

Levine, Hagai

Levy, Jonathan

Levy, Karen

Lewis, Ferdinand

Lewis, Fraser I.

Li, Fang

Li, Fang-Bai

Li, Huabin

Li, Kaigang

Li, Longjian

Li, Sen

Li, Yingxia

Li, Yonghua

Li, Zhigang
Likotrafiti, Eleni

Lim, Min K.

Lin, Chad

Lin, Hui

Lin, Kwan-Hwa

Lin, Lin

Lin, Yuan-Chung

Lincoln, Jennifer M.

Linder, Stephen

Lindsay, David

Lindström, Martin

Liu, Chi-Hung

Liu, Ching-Kuan

Liu, Hai-Ying

Liu, Hongbin

Liu, Shu

Liu, Yandi

Liu, Yang

Liu, Yaolin

Livingston, Kimberly A.

Loaiza-Bonilla, Arturo

Łobacz, Adriana

Lobb, Rebecca

Løbner-Olesen, Anders

Logue, Catherine $\mathrm{M}$.

Lonardo, Amedeo

Longo, Sonia

Lönnerfors, Celine

López-Navarro, Miguel Ángel

Loprinzi, Paul D.

Loss, Scott R.

Loughnan, Margaret

Lourenço, Roberto Wagner

Lowenstein, Lisa M.

$\mathrm{Lu}$, Hong

Lu, Jiafang

Lu, Weizhen

Lu, Xinwei

Lucchini, Sacha

Lucian Szasz, Paul

Lucio, Paulo Sergio

Łuczkiewicz, Aneta 
Luginaah, Isaac

Lukas, Michael

Luo, Zhong-Cheng

Luque, John

Lusk, Anne

Lynne, Dawkins

Lyons, Minna

MacCalman, Laura

Mackinnon, Gillian

Maggi, Stefania

Maglaveras, Nicos

Magnone, Edoardo

Magori, Krisztian

Main, Penelope

Malec, Brian

Malkhazova, Svetlana

Malliarou, Maria

Malmusia, Davide

Manaia, Célia M.

Mancianti, Francesca

Mancini, Laura

Manduca, Paola

Mangalore, Roshni

Manios, Stavros

Mannino, David M.

Mansoorc, Mohammad Azam

Marcucci, Fabrizio

Marengoni, Alessandra

Margolis, Helene

Marin, Daniela

Maritz, Gert S.

Markevych, Iana

Markides, Kyriakos S.

Marquis-Faver, Catherine

Marsiglia, Flavio

Martin, Barbara

Martinez, Cristina

Martinez, Nelda C.

Marzano, Maria

Masala, Giovanna

Masfety, Viviane Kovess

Massányi, Peter
Masuda, Naoki

Mathiesen, Amber M.

Matsuda, Shingo

Mattes, Al

Matthews, Charles

Matthies, Franziska

Matzarakis, Andreas

Maurer, John

Mawson, Anthony

May, Fiona

Mayne, Susan

Mayner, Lidia

Mazumdar, Soumya

Mcaughey, John

McBride, Graham B.

Mcclure, Peter

Mccowan, Lesley

McCoy, Sarah J. Breese

McCrossan, Brian A.

McDiarmid, Melissa A

McDonald, Ted

McDonough, Suzanne

McGuckin, Michael

McKallip, Robert J.

McKay, Ruth

McLafferty, Sara

McLellan, Deborah L.

McMillen, Robert

McNally, Richard

McPhedran, Samara

McSpirit, Stephanie

Medek, Danielle

Mehta, Tara G.

Meinke, Deanna

Melander-Wikman, Anita

Melke, Jonas

Mendenhall, Ian

Méndez, Claudio A.

Menezes-Filho, Jose Antonio

Menozzi, Davide

Menvielle, Gwenn

Metcalf, Patricia
Meyer, Elisabeth

Michaelakis, Antonios

Michelin, Marcia A.

Michimi, Akihiko

Miles, Lisa M.

Miller, Gloria

Min, Kyung Rok

Mitra, Raktim

Mlynarek, Steven

Möhner, Matthias

Moise, Imelda K.

Mollah, Kabirul A.

Monge, Susana

Mons, Ute

Monsees, Thomas

Montesano, Lius

Montoya, Ivan

Montserrat, Javier M.

Moore, Roland

Moral, Francisco J.

Morales, Aldo W.

Morency, Patrick

Moreno, Isabel María

Moriguchi, Takaya

Morin, Cory

Morleo, Michela

Morrow-Howell, Nancy

Morse, Stephen S.

Mort, Maggie

Morton, Cory M.

Moses, Wesley

Movsisyan, Narine

$\mathrm{Mu}$, Lan

Muggeo, Vito M. R.

Mughini Gras, Lapo

Muir, Susan

Muirhead, Colin

Mulla, Zuber D.

Muller, Clemma

Muller, Matthew D.

Mullins, Ray

Murase, Toshiyuki 
Murdoch, David R.

Muroga, Norihiko

Murray, Emily

Musher-Eizenman, Dara

Nadalin, Victoria

Nagaraja, T. G.

Nagelhout, Gera E.

Nagler, Rebekah H.

Nakajima, Motohiro

Nakash, Ora

Nandakumar, Kutty Selva

Nannette, Hoffman

Napoli, Christian

Narita, Daiju

Naska, Androniki

Navarro, Raúl

Neal, April P.

Nearing, Mark A.

Nemery, Benoit

Nerín, Cristina.

Neupane, Subas

Neupane, Sudan Prasad

Nicolini, Rosella

Niczyporyk, Jowita Samanta

Niedzwiedz, Claire L.

Nikaj, Silda

Nin, Jordi

Niu, Bo

Noonan, Devon

Nordell, Eva

Novak, Natalija

Null, Jan

Nunes, Raquel

Nurmatov, Ulugbek

Nyman, Anastasia

O'Hara, Marilyn

O'Malley, Patrick M.

O'Donnell, Deborah A.

O'Farrell, Patrick J.

Oberg, Anna Sara

Obregon, Maria-Jesus

Ochoa-Herrera, Valeria
Odendaal, Willem

Odland, Jon Øyvind

Oehmen, Adrian

Okechukwu, Cassandra

Olshansky, Stuart J.

Onarheim, Kristine

Ong, Corinne

Ongerth, Jerry E.

Opdam, Paul

Opfer, Sarah E.

Oppliger, Anne

Ordoñana, Juan R.

Ornelas, India

Ortega, Elena

Ortega, Rosario

Osawa, Haruki

Osborne, Katy

Oscar, Thomas

Osek, Jacek

Otsuka, Yasumasa

Otsuki, Michiko

Ottosson, Jakob Ryd

Owusu, Andrew

Paciorek, Chris

Padmanabhan, Vasantha

Paek, Jiheum

Pagán, José A.

Page, Kristen

Palacios-Ceña, Domingo

Palmiere, Cristian

Palù, Giorgio

Pampaka, Despina

Pan, Jen-Yi

Panico, Lidia

Papa, Anna

Papadopoulos, Andrew

Pappas, Steve

Park, Andrew

Park, Kyong

Partonen, Timo

Partridge, Bradley

Patil, Grete Grindal
Patnaik, Jennifer

Patwary, Masum A.

Paul, Mathilde

Paunović, Katarina

Pawlas, Natalia

Pawloski, Lisa R.

Pawłowska-Góral, Katarzyna

Payment, Pierre

Payne, Sarah

Pearce, Dora Claire

Pearce, Elizabeth N.

Pearson, Jennifer L.

Pearson, Lee

Pedersen, Charlotte Gjørup

Pedley, Stephen

Pedrotti, Jairo

Peel, Jennifer L.

Peen, Jaap

Pekmezovic, Tatjana

Penney, Tarra L.

Pennig, Sibylle

Perez-Ríos, Mónica

Perles-Roselló, María Jesús

Perry, Sara Jansen

Petersen, Arnd

Petersen, Elijah

Petersen, Eskild

Petersen, Sibylle

Petrass, Lauren

Petrosillo, Irene

Petrović, Vladimir

Pfeiler, Tamara

Pheasant, Robert

Phillips, Julie

Picano, Eugênio

Pichini, Simona

Pierce, Lisa M.

Pilgrim, David

Pillay, Viness

Pinchevsky, Gillian

Pira, Enrico

Pires, José Carlos Magalhães 
Pitkänen, Tarja

Platonov, Alexander E.

Pohanka, Miroslav

Polimeni, Antonella

Polosa, Riccardo

Pols, Jeannette

Pompili, Maurizio

Ponce, Carmen

Ponnet, Koen

Pontremoli, Roberto

Porteous, Nuala

Pośpiech, Beata

Potashkin, Judith

Pottage, Thomas

Poulsen, Henrik E.

Powers, Ann M.

Prado, Gustavo Faibischew

Prettner, Klaus

Preve, Nikolaos

Pribis, Peter

Price, Bronwyn

Principato, Maryann

Prins, Pier J. M.

Proper, Karin

Prus, Steven

Pulkki-Råback, Laura

Purkis, Steve

Purshouse, Robin

Putnam, Michelle

Qi, Feng

Qin, Hua-Peng

Quantin, Xavier

Quinn, Brian C.

Rahman, Mahfuzar

Ralph, Stephen J.

Ramos-Jorge, Maria

Ratschen, Elena

Rayner, Geof

Ready, Paul D.

Reagan, Krystle L.

Rebolj, Matejka

Reddy, Madhavi
Reefhuis, Jennita

Regidor, Enrique

Reid, Alison

Reiner, Robert C.

Reinhold, Steffen

Reisen, William K.

Ren, Wei (Vivian)

Reniers, Genserik

Rethorst, Chad D.

Reutfors, Johan

Rey, Grégoire

Rhee, Hae-Chun

Rheinberger, Christoph M.

Rhona, Julien

Riccardo, Flavia

Riccio, Angelo

Rice, Scott

Rice, Tom

Richardson, Richard

Rickard, Laura N.

Rigdon, Steve

Rijnaarts, Huub

Riley, Edward

Riordan, James

Ríos-Bedoya, Carlos F.

Roberts, Helen

Roberts, Sarah

Robinson, Gareth

Robson, Mark

Rocheleau, Gregory C.

Rochlin, Ilia

Rodda, Nicola

Rodrigues, Irene

Rodrigues, Jean-Marie

Rodríguez-Martín, Jose

Antonio

Rodriguez, Rudolph A.

Roe, Jenny

Rolle, Italia V.

Rollinson, David

Roman, Henry A.

Romero, Miguel Sánchez
Ronda-Perez, Elena

Rondinelli, Concetta

Rosário, Rafaela

Rose, Jennifer

Rosen, Barry

Rosenkranz, Richard R.

Rosenquist, Hanne

Rossignol, Daniel

Roy, Amanda

$\mathrm{Ru}$, Shaoguo

Ruano-Ravina, Alberto

Rubio, Doris McGartland

Rudatsikira, Emmanuel

Ruecker, Norma J.

Ruel, Erin

Ruiz-Bustos, Eduardo

Ruiz, Joaquim

Rull, Rudy

Russell-Mayhew, Shelly

Ruzauskas, Modestas

Ryan, Patrick H.

Sabbath, Erika

Sabri, Bushra

Saddleson, Megan

Sadler, Richard

Sáfadi, Marco A. P.

Sainz, Carlos

Sáiz, Juan-Carlos

Sakakibara, Masayuki

Salmain, Michele

Salminen, Simo

Sambrie, Vittorio

Sánchez, David

Sancho-Garnier, Helene

Sanmiquel, Lluís

Sano, Daisuke

Santos, Maria Paula

Santos, Ubiratan De Paula

Sarwer, David B.

Sass, Jennifer

Sato, Azusa

Sato, Hiroki 
Sato, Yukita

Sauleau, Erik-André

Sauni, Riitta

Savini, Giovanni

Schenk, Niels

Schillo, Barbara

Schindler, Christian

Schlomit, Paz

Schmidt, Anja

Schmitz, Norbert

Schoen, Cathy

Schoueri-Mychasiw, Nour

Schrauzer, Gerhard

Schuck, Amie

Schug, Joanna

Schultz, Annette

Schultz, Stephen

Schulz, Jennifer J.

Schuurman, Nadine

Schwaiger, Karin

Schwebel, David

Schweizer, Vanessa J.

Scialli, Anthony R.

Scotch, Matthew

Scott-Sheldon, Lori A. J.

Scott, Elizabeth

Scotton, Carol R.

Sebastiani, Paola

Seenan, Chris

Seguin, Rebecca

Sella, Luca

Selman, Carol

Selya, Arielle

Sembajwe, Grace

Sengor, Sevinc

Senthilselvan, Ambikaipakan

Serrano, Antoni

Serrano, Emilio

Setton, Eleanor M.

Sexton, Ken

Shadoin, Amy

Shakoori, Abdul Rauf
Shanahan, Fergus

Shapiro, Karen

Sheffield, Perry E.

Shen, Zhangquan

Shenawy, Nahla S. El

Shepherd, Daniel

Sherman, Recinda

Shi, Xianglin

Shi, Xun

Shiao, Yih-Horng

Shibayama, Keigo

Shiels, Aaron B.

Shiyko, Mariya

Shoff, Carla

Short, Camille E.

Shortt, Niamh K.

Shriar, Avrum J.

Siegert, Richard

Siever, Larry J.

Silbergeld, Ellen

Silley, Peter

Silva, Lígia

Simpson, Jean

Sims, Jane

Sims, Les

Sinclair, Ryan

Sintonen, Sanna

Skoczyńska, Anna

Skön, Jukka-Pekka

Skouloudis, Andreas N.

Škrbić, Biljana

Skudutyte-Rysstad, Rasa

Slaymaker, Tom

Sloan, Robert

Slotterback, Carissa Schively

Smani, Younes

Smith, Bradley

Smith, Claire

Smith, Mark

Smyrnowa, Julija

Sohl, Terry

Solcan, Carmen
Soljak, Michael

Solo-Gabriele, Helena

Son, Nguyen-Thanh

Song, Rhayun

Song, Yong

Sosa, Manuel

Sospedra, Javier

Sousa, Sofia I. V.

Sovio, Ulla

Soyer, Peter

Spangler, John

Spanò, Carmelina

Sparrow, Robert

Spector, Logan

Spengler, John O.

Srivastava, Shouryadeep

Staddon, Philip

Stadler, Hermann

Stagnitti, Karen

Stamatis, Nikolaos

Stanford, Kim

Stansbury, Kim

Stansfeld, Stephen

Stawiarska-Pięta, Barbara

Stefanelli, Dario

Stefanogiannis, Niki

Stein, Gregor

Steiner, Frederick

Steinmann, Peter

Stelzer, Jiri

Stemplewski, Rafal

Stepanov, Irina

Stichler, Jaynelle F.

Stockton, Jemima

Stokkeland, Knut

Stone, Deb

Strandh, Mattias

Strobel, Natalie

Strohmeier, Dagmar

Styers, Diane M.

$\mathrm{Su}$, Chang

Suenaga, Hikaru 
Suggs, Suzanne

Sultan, Zuraimi

Sumner, Walton Ii

Sun, Hongmin

Sun, Qinghua

Sun, Ya-Yen

Suñol, Cristina

Suominen, Tarja

Svensen, Erik

Swanberg, Jennifer E.

Sweeney, Daniel W.

Sykes, Catherine

Szabó, Szilárd

Szymańska, Jolanta

Takahashi, Masayoshi

Takeshita, Haruo

Talbert-Slagle, Kristina

Talbot, Prue

Tandlich, Roman

Tang, Zhenghong

Tasi, Su-Ying

Taylor, Sally

Tehranifar, Parisa

Telfair, Joseph

Ter Kuile, Benno

Tham, Kwok Wai

Thastum, Mikael

Theodoropoulos, Georgios

Thomas, David

Thomas, David P

Thompson, Richard E.

Thompson, Wendy

Thomson, Murray

Tian, Guoxun

Tian, Linwei

Titze, Sylvia

Tobita, Kimimasa

Todd, Ewen

Toft, Gunnar

Tokajian, Sima T.

Tol, Richard S. J.

Tommasi, Franca
Topitzes, Dimitri

Torija, Antonio J.

Toscano, William

Touboul Lundgren, Pia

Tousignant, Michel

Trail, Frances

Tralau, Catherine

Tran, Anh N.

Trasande, Leonardo

Triassi, Maria

Truax, Barry

Trudgett, Alan

Tsai, Hui-Ju

Tsai, Jack

Tsai, Perng-Jy

Tsai, Ted

Tsanas, Athanasios

Tseng, Chin-Hsiao

Tsimbos, Cleon

Tsuchiya, Kenji J.

Tu, Jun

Turell, Michael J.

Tuvesson, Hanna

Tyrväinen, Liisa

Tzelepis, Flora

Ulbert, Sebastian

Unger, Jennifer

Väisänen, Liisa

Van Beijnum, Bert-Jan

Van Broekhuizen, Pieter

Van Cauwenberg, Jelle

Van Delden, Hedwig

Van den Borst, Bram

Van Den Branden, Sigrid

Van Den Hurk, Andrew F.

Van Der Bij, Akke K.

Van Der Kooij, Dick

Van Der Poel, Wim

Van Ewijk, Reyn

Van Hoof, Joris J.

Van Kamp, Irene

Van Kippersluis, Hans
Van Kleef, Richard

Van Laer, Lut

Van Orden, Kimberly

Van Renterghem, Timothy

Van Rie, Annelies

Van Ruijven, Bas J.

Van Sluijs, Esther

Van Zandwijk, Nico

Vandebriel, Rob J.

Vandelanotte, Corneel

Vanderplasschen, Wouter

Vandoros, Sotiris

Vargo, Jason

Vaughan, Jefferson A.

Veiga, Lene

Verloigne, Maïté

Viana, Mar

Vieux, Baxter E.

Viladrich, Anahí

Vilhelmsson, Oddur

Villanueva, Vicent

Vitali, Matteo

Vlachokostas, Christos

Vollmer, Sebastian

Von Essen, Susanna

Von Mühlendahl, Karl Ernst

Von Wehrden, Henrik

Voulvoulis, Nick

Wade, Tim

Wadsworth, Daniel P.

Wahlbeck, Kristian

Walerian, Elżbieta

Walker, Ashley

Walker, Jimmy

Wallander, Jan

Wallmann-Sperlich, Birgit

Wampler, Peter

Wan, Man-Pun

Wang, Fahui

Wang, Heng-Shan

Wang, Jikun

Wang, Shengrui 
Wang, Yuanpeng

Wang, Zhong-Quan

Warburton, Darren E. R.

Ward, Michael P.

Wargocki, Pawel

Warnakulasuriya, Saman

Warren, Jim

Warren, Joshua

Warren, Paige

Warriner, Keith

Wasem, Juergen

Waters, Andrew J.

Waterworth, Eva Lindh

Watt, Toni Terling

Watts, Greg

Webb, Cameron E.

Webb, Christian

Weber, Germain

Weber, Miriam

Wei, Binnian

Weinreb, Alexander

Weinstein, Philip

Weitzman, Michael L.

Wen, Kuang-Yi

Wen, Li-Ming

Weng, Chih-Yuan

Werler, Martha

Wertheimer, Albert

Wewers, Mary Ellen

Weyandt, Lisa

Whatley, Art A.

Wherton, Joseph

While, Alison E.

White, Alan

White, Jason C.

White, Mathew

White, Sarah

Wigg, Neil

Wilhelm, Barbara
Williams, Prysor

Williams, David

Williams, Rebecca S.

Williams, Susan

Williamson, Tim

Willox, Ashlee Cunsolo

Wilson, Josephine

Wilson, Leigh

Wilson, Mark G.

Windsor, Jack

Winquist, Andrea

Withall, Janet

Wittenberg, Eve

Woc-Colburn, Laila

Wolff, Hans

Wolkoff, Peder

Wong, Bryan

Wong, Hai Ming

Wong, Tit-Yee

Woo, Yin-Tak

Woodward, Alistair

Woolford, Susan J.

Worsley, Anthony

woździak, Anna Z.

Wright, Caradee

Wu, Gang

Wu, Jianxin

Wu, Kuen-Yuh

$\mathrm{Wu}$, Xiangmei

Wunder, Christoph

$\mathrm{Xu}$, Hai

Xu, Howard

$\mathrm{Xu}$, Zhiheng

Yan, Guofen

Yan, Pingkun

Yan, Tao

Yang, Chunping

Yano, Takahashi

Yao, Weirong
Yard, Ellen E.

Yarmoliuk, Tatiana

Yaworsky, William

Yee, Don

Yeh, Gloria Y.

Yeh, Pamela J.

Yiangou, Minas

Yiannakoulias, Nikolaos

Yoons, Yoonjin

Yoshizuka, Kazuharu

You, Yaqi

Yuan, Chung-Shin

Yuan, Guo-Li

Yumak, Zerrin

Zabiegała, Bożena

Zajacova, Anna

Zander, Kerstin K.

Zegers, Doreen

Zenk, Shannon

Zhang, Jingjie

Zhang, Kai

Zhang, Ning Jackie

Zhang, Shun

Zhang, Tonglin

Zhang, Xingyou

Zhang, Xuxiang

Zhang, Yulong

Zhang, Zhiyong

Zhao, Hongtao

Zhao, Jinshun

Zheng, W. Jim

Zhou, Hongjian

Zhou, Shang-Ming

Zhou, Yibiao

Zhu, Jin Liang

Zhu, Xing-Quan

Ziegler, Matthias

Ziemińska, Elżbieta 
Zierold, Kristina

Zima, Jan
Zimmerman, Rick S.

Zotter, Jean
Zuckerman, Diana

Zvoleff, Alex

(C) 2014 by the authors; licensee MDPI, Basel, Switzerland. This article is an open access article distributed under the terms and conditions of the Creative Commons Attribution license (http://creativecommons.org/licenses/by/3.0/). 